\title{
PARECERISTAS 2015
}

\author{
Adolfo Ignacio Calderón \\ Pontifícia Universidade Católica de Campinas \\ Adriana Rocha Bruno \\ Universidade Federal de Juiz de Fora \\ Alda Junqueira Marin \\ Pontifícia Universidade Católica de São Paulo \\ Alda Regina Tognini Romaguera \\ Universidade de Sorocaba \\ Aldo Victorio Filho \\ Universidade do Estado do Rio de Janeiro \\ Alexandre Maia do Bomfim \\ Instituto Federal de Educação, Ciência e Tecnologia \\ do Rio de Janeiro
}

\section{Alexsandro da Silva}

Universidade Federal de Pernambuco

\section{Ana Ivenicki}

Universidade Federal do Rio de Janeiro

\section{Ana Luiza Bustamante Smolka}

Universidade Estadual de Campinas

Ana Maria Falcão Aragão

Universidade Estadual de Campinas

Ana Maria Villela Cavaliere

Universidade Federal do Rio de Janeiro

Ana Tereza Reis da Silva

Universidade de Brasília

Ana Waleska Pollo Campos Mendonça

Pontifícia Universidade Católica do Rio de Janeiro

Andrea Barbosa Gouveia

Universidade Federal do Paraná

Angelo Ricardo de Souza

Universidade Federal do Paraná

Antonio Carlos Rodrigues de Amorim

Universidade Estadual de Campinas

\section{Carina Elisabeth Maciel}

Universidade Federal de Mato Grosso do Sul

\section{Carlos Eduardo Ferraco}

Universidade Federal do Espírito Santo

Carlos Eduardo Vieira

Universidade Federal do Paraná

\section{Carlos Frederico Bernardo Loureiro \\ Universidade Federal do Rio de Janeiro}

Carlos Henrique Rodrigues

Universidade Federal de Santa Catarina

Carlos Herold Junior

Universidade Estadual de Maringá

Carlos Roberto Jamil Cury

Pontifícia Universidade Católica de Minas Gerais

Carmen Campoy Scriptori

Universidade Estadual de Campinas

Catarina de Souza Moro

Universidade Federal do Paraná

Célia Regina Otranto

Universidade Federal Rural do Rio de Janeiro

Célia Regina Teixeira

Universidade Federal da Paraíba

Celina Nunes de Alcântara

Universidade Federal do Rio Grande do Sul

Celso do Prado Ferraz Carvalho

Universidade Nove de Julho

Cesar Zucco

Universidade Federal de Santa Catarina

Claudia Maria de Lima

Universidade Estadual Paulista

Cláudia Ribeiro Bellochio

Universidade Federal de Santa Maria

Cláudio Almir Dalbosco

Universidade de Passo Fundo

Cleonice Maria Tomazzetti

Universidade Federal de São Carlos

Cristina Carvalho

Pontifícia Universidade Católica do Rio de Janeiro

Dalila Andrade Oliveira

Universidade Federal de Minas Gerais

David Mesquita da Costa

Centro Universitário Adventista de São Paulo

Delcele Mascarenhas Queiroz

Universidade do Estado da Bahia 
Denise de Freitas

Universidade Federal de São Carlos

Dilma Antunes Silva

Prefeitura de São Paulo

Dirce Djanara Pacheco e Zan

Universidade Estadual de Campinas

Doris Pires Vargas Bolzan

Universidade Federal de Santa Maria

Edméa Oliveira dos Santos

Universidade do Estado do Rio de Janeiro

Edna Maria Querido de Oliveira Chamon

Universidade de Taubaté

Edna Mendonça Oliveira de Queiroz

Universidade Federal de Goiás

Eduardo Paiva de Pontes Vieira

Universidade Federal do Pará

Eliana Borges Correia de Albuquerque

Universidade Federal de Pernambuco

Elianda Figueiredo Arantes Tiballi

Pontifícia Universidade Católica de Goiás

Elizabeth de Lima Gil Vieira

Universidade Federal do Estado do Rio de Janeiro

Emília Freitas de Lima

Universidade Federal de São Carlos

Erisvaldo Pereira dos Santos

Universidade Federal de Ouro Preto

Eugênio Paccelli Aguiar Freire

Universidade Federal do Rio Grande do Norte

Fabiana de Amorim Marcello

Universidade Federal do Rio Grande do Sul

Fabiany de Cássia Tavares Silva

Universidade Federal de Mato Grosso do Sul

Fabrícia Teixeira Borges

Universidade de Brasília

Fernando Henrique Protetti

Instituto Federal de Educação, Ciência e

Tecnologia de São Paulo

Flávia Brocchetto Ramos

Universidade de Caxias do Sul

Flávia Inês Schilling

Universidade de São Paulo

Flavinês Rebolo

Universidade Católica Dom Bosco

Georgia Sobreira dos Santos Cêa

Universidade Federal de Alagoas
Gilda Helena Bernardino de Campos

Pontifícia Universidade Católica do Rio de Janeiro

Gisely de Nazaré Freitas da Silva

Universidade Federal do Pará

Graziela Giusti Pachane

Universidade Federal do Triângulo Mineiro

Guaracira Gouvêa de Sousa

Universidade Federal do Estado do Rio de Janeiro

Gustavo Enrique Fischman

Arizona State University

Iara Tatiana Bonin

Universidade Luterana do Brasil

Irma Rizzini

Universidade Federal do Rio de Janeiro

Inês Ferreira de Souza Bragança

Universidade do Estado do Rio de Janeiro

Isabel Cristina Moura Carvalho

Pontifícia Universidade Católica do Rio

Grande do Sul

Itale Luciane Cericato

Universidade Federal de São Paulo

Ivana Maria Schnitman

Centro Universitário Estácio da Bahia

Jadir de Morais Pessoa

Universidade Federal de Goiás

Joana Peixoto

Pontifícia Universidade Católica de Goiás

Joice de Espindola

Universidade Federal de Pernambuco

José Adelson da Cruz

Universidade Federal de Goiás

Jose Armando Valente

Universidade Estadual de Campinas

José Geraldo Silveira Bueno

Pontifícia Universidade Católica de São Paulo

Juan Rolando Cornejo

Universidad Católica del Maule

Julio Roberto Groppa Aquino

Universidade de São Paulo

Karla Schuck Saraiva

Universidade Luterana do Brasil

Laura Cristina Vieira Pizzi

Universidade Federal de Alagoas

Lílian de Aragão Bastos do Valle

Universidade do Estado do Rio de Janeiro 
Livia da Conceição Costa Zaqueu

Universidade Presbiteriana Mackenzie

Lucídio Bianchetti

Universidade Federal de Santa Catarina

Luciola Licínio de Castro Paixão Santos

Universidade Federal de Minas Gerais

Luis Felipe Carvalho

Pontifícia Universidade Católica do Rio de Janeiro

Luís Filipe Barbeiro

Instituto Politécnico de Leiria

Luís Henrique Sommer

Universidade Federal do Rio Grande do Sul

Luiz Artur dos Santos Cestari

Universidade Estadual do Sudoeste da Bahia

Luiz Caldeira Brant de Tolentino Neto

Universidade Federal de Santa Maria

Marco Antonio Leandro Barzano

Universidade Estadual de Feira de Santana

Marcos Augusto Souza Rodrigues da Silva

Universidade Camilo Castelo Branco

Marcos Cezar de Freitas

Universidade Federal de São Paulo

Marcos Freisleben Zorzal

Universidade Federal de São Carlos

Marcos José da Silveira Mazzotta

Universidade de São Paulo

Mareli Eliane Graupe

Universidade do Planalto Catarinense

Maria Amélia da Costa Lopes

Universidade do Porto

Maria Cecília Lorea Leite

Universidade Federal de Pelotas

Maria Cristina de Soares Gouvêa

Universidade Federal de Minas Gerais

Maria Cristina Piumbato Innocentini Hayashi

Universidade Federal de São Carlos

Maria da Conceição Passeggi

Universidade Federal do Rio Grande do Norte

Maria da Penha Casado Alves

Universidade Federal do Rio Grande do Norte

Maria de Fátima Teixeira Barreto

Universidade Federal de Goiás

Maria Dilnéia Espindola Fernandes

Universidade Federal de Mato Grosso do Sul
Maria do Carmo de Lacerda Peixoto

Universidade Federal de Minas Gerais

Maria do Carmo Martins

Universidade Estadual de Campinas

Maria dos Remédios de Brito

Universidade Federal do Pará

Maria Isabel da Cunha

Universidade do Vale do Rio dos Sinos

Maria Isabel Ramalho Ortigão

Universidade do Estado do Rio de Janeiro

Maria Luiza Canedo Queiroz da Silva

Pontifícia Universidade Católica do Rio de Janeiro

Maria Luísa Frazão Branco

Universidade da Beira Interior

Maria Luiza Süssekind Verissimo Cinelli

Universidade Federal do Estado do Rio de Janeiro

Maria Manuela Alves Garcia

Universidade Federal de Pelotas

Maria Rita de Almeida Toledo

Universidade Federal de São Paulo

Marilda Oliveira de Oliveira

Universidade Federal de Santa Maria

Marilia Gouvea de Miranda

Universidade Federal de Goiás

Marilyn Aparecida Errobidarte de Matos Instituto Federal de Educação, Ciência e

Tecnologia de Mato Grosso do Sul

Mário Luiz Neves de Azevedo

Universidade Estadual de Maringá

Marlene Ribeiro

Universidade Federal do Rio Grande do Sul

Marly Amarilha

Universidade Federal do Rio Grande do Norte

Martha Marandino

Universidade de São Paulo

Maurício Rosa

Universidade Federal do Rio de Janeiro

Monica Fantin

Universidade Federal de Santa Catarina

Neil Franco Pereira de Almeida

Universidade Federal de Mato Grosso

Nelson Cardoso Amaral

Universidade Federal de Goiás

Nerli Nonato Ribeiro Mori

Universidade Estadual de Maringá 


\author{
Neusa Salim Miranda \\ Universidade Federal de Juiz de Fora \\ Pablo Silva Machado Bispo dos Santos \\ Universidade Federal Fluminense \\ Paulo da Silva Gusmão \\ Universidade Federal de Santa Maria \\ Pedro Roberto Jacobi \\ Universidade de São Paulo \\ Rejane Cavalheiro \\ Universidade Federal de Santa Maria \\ Renato José de Oliveira \\ Universidade Federal do Rio de Janeiro \\ Renato Monteiro Athias \\ Universidade Federal de Pernambuco \\ Rita Maria Decarli Bottega \\ Universidade Estadual do Oeste do Paraná \\ Ronaldo Marcos de Lima Araújo \\ Universidade Federal do Pará \\ Rosa Maria Bueno Fischer \\ Universidade Federal do Rio Grande do Sul

\section{Rosália Maria Duarte \\ Pontifícia Universidade Católica do Rio de Janeiro \\ Rosângela de Amorim Teixeira de Oliveira Instituto Federal Catarinense \\ Rosângela Tenório de Carvalho \\ Universidade Federal de Pernambuco} \\ Rosemary Dore Heijmans \\ Universidade Federal de Minas Gerais \\ Rosemeire Reis da Silva \\ Universidade Federal de Alagoas \\ Rute Estanislava Tolocka \\ Universidade Metodista de Piracicaba
}

Sabrina Moeblecke

Universidade Federal do Rio de Janeiro

Sandra Regina Sales

Universidade Federal Rural do Rio de Janeiro

Sandra Valéria Limonta Rosa

Universidade Federal de Goiás

Sérgio Paulino Abranches

Universidade Federal de Pernambuco

Silke Weber

Universidade Federal de Pernambuco

Silvia Helena Vieira Cruz

Universidade Federal do Ceará

Simone Torres Evangelista

Universidade Federal Fluminense

Sirlei de Lourdes Lauxen

Universidade de Cruz Alta

Soely Aparecida Jorge Polydoro

Universidade Estadual de Campinas

Sônia Cristina Soares Dias Vermelho

Universidade Federal do Rio de Janeiro

Theresa Maria de Freitas Adrião

Universidade Estadual de Campinas

Vera Lúcia Gaspar da Silva

Universidade do Estado de Santa Catarina

Vera Maria Nigro de Souza Placco

Pontifícia Universidade Católica de São Paulo

Wellington Pereira das Virgens

Universidade Federal de São Paulo

Wilsa Maria Ramos

Universidade de Brasília

Carlos Eduardo Vieira

Universidade Federal do Paraná, Curitiba, PR, Brasil

Antonio Carlos Rodrigues de Amorim

Universidade Estadual de Campinas, Campinas, SP, Brasil

Cláudia Ribeiro Bellochio

Universidade Federal de Santa Maria, Santa Maria, RS, Brasil 


\section{Laura Cristina Vieira Pizzi}

Universidade Federal de Alagoas, Maceió, AL, Brasil

Marcelo Andrade

Pontifícia Universidade Católica do Rio de Janeiro, Rio de Janeiro, RJ, Brasil

Maria da Conceição Passeggi Universidade Federal do Rio Grande do Norte, Natal, RN, Brasil

Marília Gouvea de Miranda

Universidade Federal de Goiás, Goiânia, GO, Brasil

Rio de Janeiro, janeiro de 2016 